\title{
CDISC SEND Pregnancy Findings Test Name Terminology
}

National Cancer Institute

\section{Source}

National Cancer Institute. CDISC SEND Pregnancy Findings Test Name Terminology. NCI

Thesaurus. Code C124325.

Terminology associated with the pregnancy findings test name codelist of the Clinical Data Interchange Standards Consortium (CDISC) Standard for the Exchange of Nonclinical data (SEND). 